\title{
Topological Features Detection and Automatic Segmentation of Point Clouds from Tree Surface
}

\author{
H. Liu, H.Q. Zhang, H.B. Ju \\ Research Institute of Forestry Resource Information Techniques \\ CAF, China
}

\begin{abstract}
In this paper, the tree topological features detection and automatic segmentation of point cloud data are studied, and propose a method of topological features detection and automatic segmentation of point cloud data, based on panoramic range image. First of all, the point cloud data is converted into the cylindrical polar coordinates and create the panoramic range image of trees. Then, according to the level of the different structural characteristics can be automatic segmentation trees. These feature points trees point cloud can be divided into two parts of the tree trunks and branches structure. The experimental results show that the method can improve the efficiency and accuracy of the topological features detection and automatic segmentation of the point clouds.
\end{abstract}

Keywords-point cloud data; panoramic range image; automatic segmentation

\section{INTRODUCTION}

The tree simulation is a very important component in the field of computer graphics and virtual reality [1]. Especially creating and rendering of tree models and computer animation, those are widely used in advertisement, animated cartoon, film, computer design, education, military etc. At the first, the trees have variety and structural complexity constituted by trunk, branches and leaves in the reality [2]. The second, the computing power of the computer now is limited. The third, 3D model processing takes large memory of the computer. All of above, the tree simulation is difficult to progress. In the meantime, trees have the typical branch structure. If we can extract the tree topology feature points, then connect these feature points form a trees frame [3-8]. It can effectively reflect the shape of the trees and the topological connection, and can greatly simplify the geometric representation of the trees. Thus, quickly and accurately extract the topological feature points of trees is of great significance [9].

\section{ESTABLISH PANORAMIC RANGE IMAGE OF POINT CLOUD DATA FROM TREE SURFACE}

In this paper we use FARO 3D laser scanner to gain point cloud data of branch structure relatively complete scan single-column trees. Each point in the point cloud data contains $3 \mathrm{~d}$ coordinate $(\mathrm{x}, \mathrm{y}, \mathrm{z})$ and colour values $(\mathrm{R}, \mathrm{G}, \mathrm{B})$, and using Cartesian space rectangular coordinate system as a standard coordinate system. Because the trees can be regard as a cylindrical structure, thus we can establish proper cylindrical symmetry axis coordinate system according to the trees, the Cartesian coordinates of the point cloud data can be converted into cylindrical coordinates. Based on the above reasons, the trees a panoramic view of range image can be obtained. The whole process is divided into the following three steps:
1. To establish a cylindrical coordinate system of tree point cloud;

2. To transform the Cartesian coordinates $(\mathrm{x}, \mathrm{y}, \mathrm{z})$ of the point cloud data into cylindrical coordinates $(\rho, \theta, \mathrm{z})$;

3. In cylindrical coordinate system, using $\theta$ as the abscissa, $\mathrm{z}$ as the ordinate, $\rho$ as the depth values, to generate panoramic range image $(\theta, \mathrm{z}, \rho)$.

\section{A. Point cloud Data in Cylindrical Coordinate System}

Firstly calculates the minimum point cloud trees surrounded by columns, then with the minimum bounding cylinder bottom as a cylindrical coordinate system $\mathrm{x}$ and $\mathrm{y}$ plane, cylindrical symmetry axis as the $\mathrm{Z}$ axis, the direction of the upward as the positive direction, the projection point of the axis in the XY plane as a cylindrical coordinate system origin of coordinates, finally established trees point cloud the surface of the cylindrical coordinate system (figure 1).

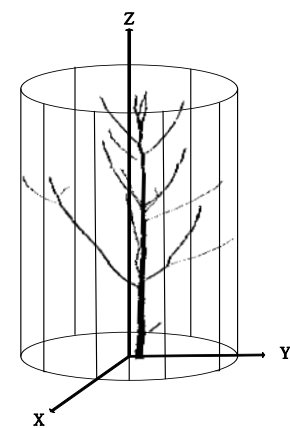

FIGURE I. THE CYLINDRICAL COORDINATE SYSTEM OF TREE POINT CLOUD DATA.

\section{B. Coordinate System transformation}

Cylindrical coordinate system is a kind of three-dimensional coordinate system. According to the formula 1 to make the association between cylindrical coordinate system and Cartesian coordinate system, we can get the trees on the surface of cylindrical coordinate form of point cloud data.

$$
\left\{\begin{array}{l}
\rho=\sqrt{x^{2}+y^{2}} \\
\theta=\arctan \left(\frac{y}{x}\right) \\
z=z
\end{array}\right.
$$

As shown in figure 2, cylindrical coordinates of point $\mathrm{P}$ is 
$(\rho, \theta, \mathrm{z}) . \quad \rho$ is the vertical distance of the point $\mathrm{p}$ and the $\mathrm{z}$ axis. The angle $\theta$ is the angle between line OP project to a line in the $\mathrm{x}, \mathrm{y}$ plane and the $\mathrm{x}$ axis, which range from 0 to $2 \pi$. The value $\mathrm{z}$ keep not change, and its scope is 0 to $R^{+}$.

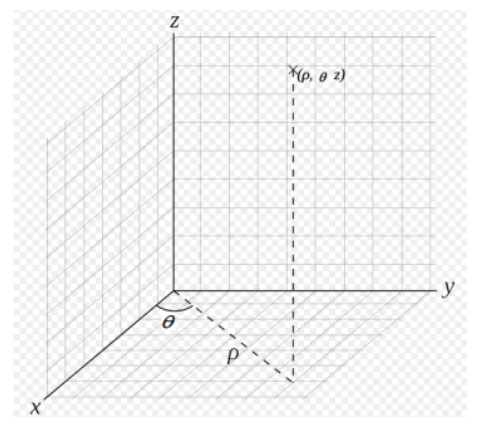

FIGURE II. CYLINDRICAL COORDINATE.

\section{Panoramic Range Image Creation}

Cylindrical coordinates of the point cloud data can be expressed as $(\rho, \theta, \mathrm{z})$. If we set $\rho$ as the $(\theta, \mathrm{z})$ function value, then, take $\theta, \mathrm{z}$ as abscissa and ordinate respectively, $\rho$ as the depth value of the $(\theta, \mathrm{z})$, we can establish the panoramic range image $(\theta, \mathrm{z}, \rho)$. The resulting panoramic range image is shown in figure 3.

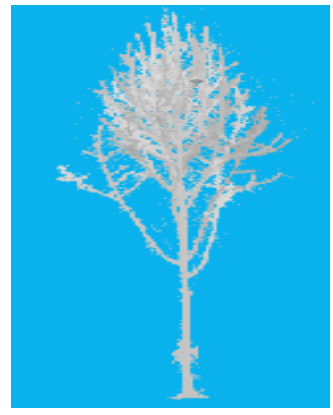

FIGURE III. PANORAMIC RANGE IMAGE OF TREE.

\section{AUTOMATIC SEGMENTATION OF THE POINT CLOUDS FROM TREE SURFACE BASED ON PANORAMIC RANGE IMAGE}

As you can see in figure 3, different trees have different levels of structure information. For the standard trees scanning surface, we can accurately detect the key characteristics of the tree trunks and branches by using hierarchical information of the panoramic range image.

\section{A. Hierarchy Analysis}

Fitting the panoramic range image on the surface of the tree by using the least squares method [10-14]. As shown in figure 3 , different branch structure in its corresponding image domain contains some different levels on the surface. It is observed that the number of layers with the rays pass through obvious change in outstanding feature points and the critical point as we see in figure 4 . Trunk area contains only a layer of surface, primary branches area contains three layer surfaces and secondary branches area can contain three to five layers surface.
From the figure 4 , we come to the following conclusions that levels will be increased in ramification points and levels will be reduced in extreme points. Based on the above reasons, we can determine the feature points and the critical points by the tendency of the hierarchy.

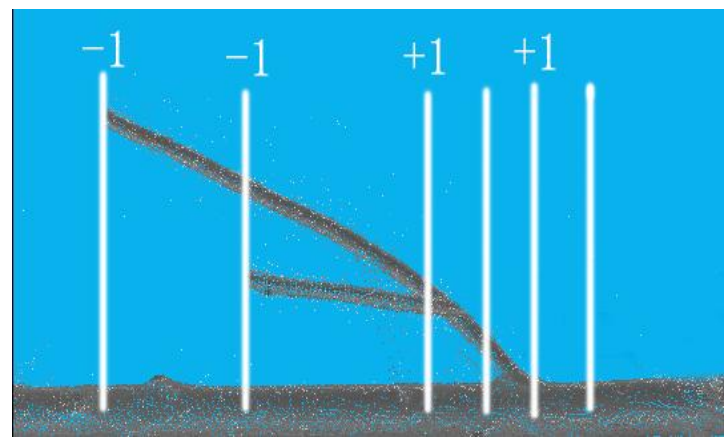

FIGURE IV. THE HIERARCHICAL INFORMATION OF PANORAMIC RANGE IMAGE FROM PART OF THE TREE.

\section{B. Topological Features Detection}

Using the level of the different structural characteristics can be automatic segmentation trees by the trees panoramic range image. Scanning image domain of panoramic range image in the segmentation, according to the number of level features containing trees feature point detection and critical point of the pixel units. Then, to extract the key characteristics of the pixel region on the point cloud processing, make the point cloud as projection rays in the image domain and write down times of each point cloud data across the surfaces. Finally we can accord to the change trend of point cloud layer information, to find a key characteristic of data points. After found the key feature points and panoramic image segmentation in the trees, we can through the coordinate transformation and the key characteristics of $3 \mathrm{~d}$ point cloud surface of trees, and according to the characters of key points to realize segmentation to the trees. The algorithm is as following:

1. Step 1, according to the 2D K-D tree structure to search depth domain image pixels, which level changed and mark those pixels. Increase to +1 , reduced to -1 .

2. Step 2, extracting the key characteristics of the pixel 
region on the point cloud processing, make the point cloud as projection rays in the image domain and write down times of each point cloud data across the surfaces. If the layer increases, the notes for +1 , on the contrary, down to -1 .

3. Step 3, looking for pairs of $(+1)$ and $(-1)$, conversion key characteristics of the coordinates of the point [ $\theta_{, \mathrm{z},} \rho_{\text {] }}$ aim to calculate coordinates of the key feature points in the Cartesian coordinate system. Then, according to key features in the trees of the scanning surface, realize the characteristics of the tree points cloud segmentation.

\section{THE APPLICATION RESULTS}

Figure 5(a) is the panoramic depth image, which in accordance with the above algorithm after point cloud data segmentation. Figure 5(b) is the structure of the tree trunks and branches key feature points, use these feature points trees point cloud can be divided into two parts of the tree trunks and branches structure.

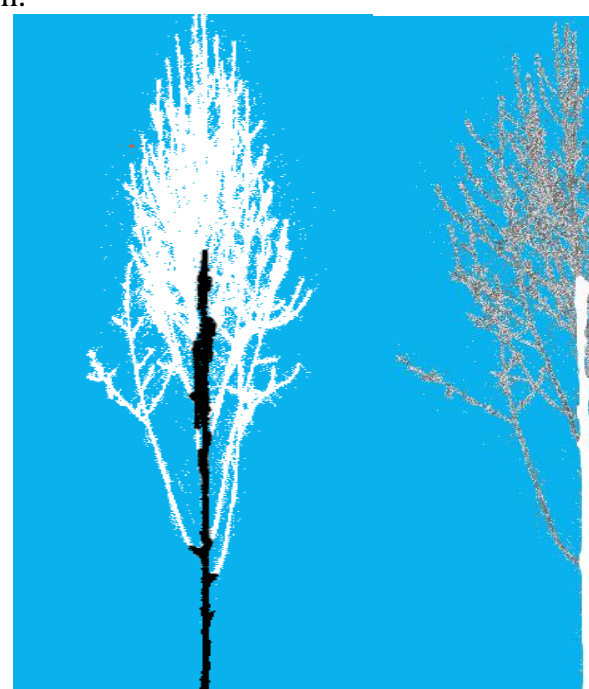

(a)

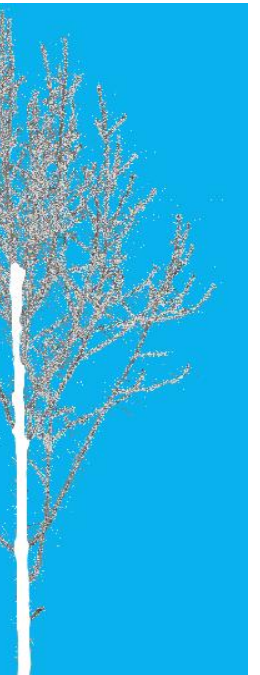

(b)

FIGURE V. (A) IS THE SEGMENTATION RESULTS OF PANORAMIC RANGE IMAGE, (B) IS THE S EGMENTATION RESULTS OF POINT CLOUD DATA.

According to the above method, extraction of height of point cloud data from trees and comparing with the actual measurement data, see table 1 . We select 10 trees on the error analysis, trees ID from 1 to 10 . Relative error is around 5\% compared with measured data; so the feasibility and effectiveness of the quantitative method are confirmed. The result shows that the key feature points segmentation give a good precision and the trees point cloud segmentation quality is well.

TABLE I. PARAMETER COMPARISON RESULTS.

\begin{tabular}{|c|c|c|c|c|c|c|c|c|c|c|}
\hline ID & 1 & 2 & 3 & 4 & 5 & 6 & 7 & 8 & 9 & 1 \\
\hline $\begin{array}{l}\text { actual } \\
\text { measureme } \\
\text { nt(m) }\end{array}$ & $\begin{array}{l}7 . \\
1\end{array}$ & $\begin{array}{l}8 . \\
9\end{array}$ & $\begin{array}{l}11 . \\
0\end{array}$ & $\begin{array}{l}9 . \\
3\end{array}$ & $\begin{array}{l}10 . \\
9\end{array}$ & $\begin{array}{l}10 . \\
1\end{array}$ & $\begin{array}{l}10 . \\
4\end{array}$ & $\begin{array}{l}11 . \\
9\end{array}$ & $\begin{array}{l}8 . \\
3\end{array}$ & $\begin{array}{l}9 . \\
0\end{array}$ \\
\hline $\begin{array}{l}\text { scanning } \\
\text { measureme } \\
\text { nt(m) }\end{array}$ & $\begin{array}{l}7 . \\
5\end{array}$ & $\begin{array}{l}9 . \\
3\end{array}$ & $\begin{array}{l}11 . \\
4\end{array}$ & $\begin{array}{l}9 . \\
8\end{array}$ & $\begin{array}{l}11 . \\
5\end{array}$ & $\begin{array}{l}10 . \\
5\end{array}$ & $\begin{array}{l}10 . \\
9\end{array}$ & $\begin{array}{l}12 . \\
6\end{array}$ & $\begin{array}{l}8 . \\
9\end{array}$ & $\begin{array}{l}9 . \\
4\end{array}$ \\
\hline $\begin{array}{l}\text { error } \\
\text { range }(\%)\end{array}$ & $\begin{array}{l}5 . \\
3\end{array}$ & $\begin{array}{l}4 . \\
3 \\
\end{array}$ & 3.5 & $\begin{array}{l}5 . \\
1\end{array}$ & 5.2 & 3.8 & 4.6 & 5.6 & $\begin{array}{l}6 . \\
7\end{array}$ & $\begin{array}{l}4 . \\
3\end{array}$ \\
\hline
\end{tabular}

\section{CONCLUSION AND DISCUSSION}

This paper present a method for automatic segmentation point cloud data based on the panoramic range image of the trees. This method is not sensitive to non-uniform distribution of point cloud data and is insensitive to noise. This article uses the panoramic depth image to represent the scattered point cloud data of the trees, by using the method of polar coordinates conversion, these for tree reconstruction of point cloud data simplification, curvature properties calculation; division of tree structure and high density mass point cloud data compression and transmission of research is meaningful. In general, the method adopted in this paper can rapidly extract trunk and branches of the trees. The next step of work will be focused on the extraction of individual branches.

\section{ACKNOWLEDGMENT}

The paper was supported by National High Technology Research and Development Program 863 (2012AA102002) and National Natural Science Foundation of China (31170590). The paper's corresponding author is Haying ZHANG.

\section{REFERENCES}

[1] Gao Shizeng, Zhang Huaiqing.\& LiuMin, Morphological Parameters Extraction of Tree Branches Based on Point Cloud. Journal of Northeast Forestry University, 42(4), pp. 109-114, 2014.

[2] Yang Meng.\&Wu Enhua, Self-Adapting Algorithm of 3D Art-Designing Tree Skeleton Extraction Journal of Frontiers of Computer Science and Technology, 6(11), pp. 1039-1048,2012.

[3] HuangHongyu,ChenChongcheng.\& ZouJie,Tree Geometrical 3D Mode ling from Terrestrial La-ser Scanned Point Clouds:A Review.Scientia S ilvae Sinicae, 49(4), pp.123-130,2013.

[4] ZhangQiyong, CenMinyi.\&ZhouGuoqing,Extracting Trees from LiDA $\mathrm{R}$ Data in Urban Region.Acta Geodaetica et Cartographica Sinica, 38(4), pp. 330-335,2008. 
[5] ZhouYi.\& ZhouGusheng,STUDY ON PROCESSING AND EXTRAC TING METHODS FOR 3D GIS DATA-THE EXAMPLE OF EXTR ACTING TREES FROM CLOUD DATA.

Computer Applications and Software, 22(10), pp. 249-250,2010.

[6] LiuFeng.\&YangZhigao,Identification of urban vegetation with laser po int cloud data based on object.Journal of Central South Forestry Univer sity,30(7), pp. 73-77,2010.

[7] Liu Hongwei.\& ShaoChanghao,Research Status and Prospect of 3D Gr een Quantity Based on the Data of 3D Laser Scanner

Journal of Anhui Agricultural Sciences, 38(24), pp. 13253-13254,2010.

[8] Cheng Zhanglin, Yan Feilong. \& Chen Baoquan, Texture - Lobes for Tree Modelling.

Journal of Computer-Aided Design \& Computer Graphics, 24(1), pp. 2-4,2012.

[9] Gao Shizeng, Zhang Huaiqing.\& LiuMin,Contour extraction of point $\mathrm{cl}$ oud data for tree branches.Journal of Zhejiang Forestry College, 30(5), pp. 648-654,2013.

[10] Gao Ziqiang.\& Wang Zhongren, A Study of Extraction and Modeling of Three-Dimensional La-ser Scanning of Tree Trunk. GEOMATICS \& SPATIAL INFORMATION TECHNOLOGY, 35(7), pp. 67-73, 2012.

[11] Ye Wen, Yun Ting.\& Ye Ning, Local surface fitting and topology structure based on timber trunk of point cloud. JOURNAL OF SHANDONG UNIVERSITY (ENGINEERING SCIENCE), 43(2),pp. 42-47,2013.

[12] Gao Shizeng, Zhang Huaiqing.\& Liu Min,Constructing Technology of Tree Branches Delaunay Triangulatio n.Journal of Southwest Forestry College, 33(3), pp. 62-68,2013.

[13] LiDongfang,Least Square Estimate of the Partial Derivatives and its S AS Procedure. Jounal of Xichang College, 28(1), pp. 36-38,2014.

[14] XiongJunhua, FangYuanmin.\& DengDebiao,Surface fitting filtering ba sed on least square method from LiDAR data.Science of Surveying and Mapping, 38(4), pp. 74-76,2013. 